\title{
Comparing the Effects of Native and Standard Strains of Monascus Purpureus on Fat Metabolism in Rats
}

\author{
Marzieh Rezaei, Rasoul Roghanian*, Iraj Nahvi and Jamal Moshtaghian
}

Department of Biology, Faculty of Sciences, University of Isfahan, Isfahan, Iran

\begin{abstract}
Background: Monascus purpureus (MP) is a microscopic fungus that belongs to the class of Ascomycets. It has a wide range of use as in pigmenting, flavoring and producing preservative agents for food stuffs as well as cholesterol-lowering agents in medications. This study was designed to compare the effects of a native MP isolated from the microbial collection at the University of Isfahan in Iran and DSM1603, the standard strain on alterations in concentration levels of cholesterol (Chol), triglyceride (TG), low density lipoprotein (LDL) and high density lipoprotein (HDL) in treated rats sera.
\end{abstract}

Methods: Pigments from the two strains were produced through submerged fermentation. 25 Wistar rats with a mean body weight of 250 grams were distributed into 5 groups of 5 each. Group 1 and Group 2 received red native pigment with concentrations of either $25 \%$ or $100 \%$ respectively. Group 3 and Group 4 received red standard pigment with concentrations of either $25 \%$ or $100 \%$ respectively. Treated animals had only free access to pigment solutions as drinking while animals in the control group had only free access to regular drinking water.

Results: The results indicate that using pigment in the diet of rats routinely could decrease the concentration levels of Chol, TG and LDL but increase HDL. In this study by using optimization of the culture medium, no adverse effect was observed in the treated animal comparing with the control group.

Conclusion: Comparing the effects of the two strains, similar results were observed but the native strain was more effective in and the one were the same in increasing HDL concentration.

Keywords: Monascus purpureus; Pigment; Rat; Hyperlipidem

Abbreviations: MP (Monascus porpureus), Chol (cholesterol), LDL-C (Low Density Lipo Protein cholesterol), HDL-C (High Density Lipo Protein cholesterol), TG (Triglyceride), HMG-CoA reductase (3-hydroxy-3-methylglutaryl coenzyme A reductase), CVRF (cardiovascular risk factors), STD Monascus purpureus (Standard, DSM1603).

\section{Introduction}

Cholesterol (Chol) is a required blood lipid for the body maintenance in a reasonable amount. However, high cholesterol levels may cause atherosclerosis or other cardiovascular diseases which could lead to heart attack. All types of cardiovascular diseases are now among the main factors causing disabilities and mortalities in developed countries. There are many cardiovascular risk factors (CVRF) involved in cardiovascular diseases of which the most important one is unusual high level of blood cholesterol. The extensive epidemiological studies have revealed a direct correlation between atherosclerosis and total blood Chol levels particularly the cholesterol bound to low density lipoproteins (LDL).

Controlling Chol levels and the improved methods for increasing good cholesterol or lowering bad cholesterol levels could be an important step to lowering risks in people susceptible to cardiovascular diseases. Mostly, modifications in lifestyle may be good enough to normalize Chol levels. Such modifications can include dietary changes, moderate exercise, and regular use of antioxidants and other cholesterol lowering agents such as pigments, fibroses, nicotinic acids and bile acid resins. There is an obvious need for further researches despite progresses in finding new compounds and developing methods to lower human blood TC and LDL to reduce CVRF. Comparing the effect of a native Monascus purpureus or more commonly known as red mold rice isolated from the microbial collection at University of Isfahan in Iran with the effect of the standard strain, DSM1603 on alterations in concentration levels of cholesterol (Chol), triglyceride (TG), low density lipoprotein (LDL) and high density lipoprotein (HDL) in treated rats was the basic idea of designing this study.

The important characteristics of MP play significant roles in the human lifestyle as well as in the environment. This fungus produces pigments through its fermentation which have been traditionally used as additive, coloring or flavoring agents in foods and beverages in China $[1,2]$. The metabolites obtained from MP comprise six known azaphilone pigments with a color spectrum ranging from yellow to red [3]. These pigments have various biological properties including hypolipidemic [4], antitumor promotion [5], immunosuppressive [6] and plasma glucose-lowering [7]. In addition, the pigments contain some antioxidant compounds including dimerumic acid [8], 3-hydoxy4-methoxy-benzoic acid [9] and dihydromonacolin-MV [10].

MP naturally forms 3-hydroxy-3-methylglutaryl coenzyme A reductase (HMG-CoA reductase) which is an inhibitor known as

*Corresponding author: Dr. Rasoul Roghanian, Department of Biology, Faculty of Sciences, University of Isfahan, Hezarjerib St., Isfahan, Iran, Postal Code: 81746 73441 Tel: +98311-7932458; Fax: +98311-7932456; E-mail: Rasoul_roghanian@ yahoo.co.uk

Received November 11, 2011; Accepted December 13, 2011; Published December 15, 2011

Citation: Rezaei M, Roghanian R, Nahvi I, Moshtaghian J (2011) Comparing the Effects of Native and Standard Strains of Monascus Purpureus on Fat Metabolism in Rats. J Bioequiv Availab 3: 258-262. doi:10.4172/jbb.1000096

Copyright: $\odot 2011$ Rezaei M, et al. This is an open-access article distributed under the terms of the Creative Commons Attribution License, which permits unrestricted use, distribution, and reproduction in any medium, provided the original author and source are credited. 
monacolin and commercially recognized as Mevacor, Cholestin, Lovastatin, Statin etcetera. Antioxidant effect of statin is quoted to be beyond atherosclerosis beneficent with the potential therapeutic advantages for atrial fibrillation and heart failure [11]. Monacolin K is a potent inhibitor of HMG-CoA reductase, the enzyme involved in the process of cholesterol biosynthesis. It has the potency to maintain a healthy blood lipid level by means of decreasing the production of cholesterol in human. MP pigments favorably influence lipid profiles in hypercholesterolemic patients. As the alternative treatments, they may be of great value in prevention and treatment of hypercholesterolemia and atherosclerosis [12-18].

Another application of MP is to extract and purify its pigments as food coloring substances. In order to enhance meat color, several authors [19-22] have used MP pigments as substitutes for nitrate/ nitrate salts. It is reported that poultry and ham prepared with half the quantity of nitrite salting mixture of Monascus purpureus extract maintained the most desirable color, flavor and appearance as well as the best microbiological parameters along with the most suitable salt content [23]. A novel formulation approach to prepare nanoparticulate red mold rice is also reported with both in vitro and in vivo safety evaluations indicating no mutagenic or toxic responses [24]

\section{Methods}

\section{Microorganism and pigment production}

Submerged fermentation was the cultivation method applied in this study. The native MP and the standard one, DSM1603 were cultivated on slanted malt extract agar (MEA) at $25^{\circ} \mathrm{C}$. Then, the seed cultures were prepared by transferring a loop full of spore from MEA agar slanted into a 500-ml flask containing $100 \mathrm{ml}$ basal medium containing $5 \mathrm{~g} / \mathrm{l}$ peptone, $3 \mathrm{~g} / \mathrm{l}$ malt extract and $10 \mathrm{~g} / \mathrm{l}$ glucose with the $\mathrm{pH}$ set at 5.0. Each $500 \mathrm{ml}$ Erlenmeyer flask containing $80 \mathrm{ml}$ of the produced medium (semi-synthetic) was incubated with $20 \mathrm{ml}$ of the inoculums culture and incubated at $30^{\circ} \mathrm{C}, 300 \mathrm{rpm}$ for 64 to 72 hours [25]. The fermentation process was carried out under the conditions of $\mathrm{pH}$, 6-7; agitation, $200-300 \mathrm{rpm}$; temperature, $25-30^{\circ} \mathrm{C}$ for a period of 5-7 days [26-27]. Pigments were released into the fermentation medium through mycelia [28]. The fermentation broth medium containing red pigment was separated from the mycelia using Watman filter No 1 [25]. The filtrated broth was used instead of drinking for the treated rats or it was diluted using clean tap water to prepare lover dose of the pigment (i.e. 25\%).

\section{Rats and pigment treatment}

25 male Wistar rats, 8 weeks of age with an average body weight of $250 \mathrm{~g}$, were obtained from the laboratory animal unit of Chamran University, Ahvaz, Iran. The animals were housed in standard plastic cages and kept in a room under standard conditions of temperature, $22 \pm 2^{\circ} \mathrm{C}$; hydrometry, $50 \pm 5 \%$; 12 -hour reverse light-dark cycle and free access to regular rat chow pellets and water. After 2 weeks of accommodation, the animals were semi-randomly distributed into 5 groups of 5 each as indicated in Table 1. Group 1 and Group 2 received red native pigment with the concentrations of either $25 \%$ or $100 \%$ respectively. Group 3 and Group 4 received red standard pigment with concentrations of either $25 \%$ or $100 \%$ respectively. Treated animals had only free access to pigment solutions as drinking while animals in the control group had only free access to regular drinking water.

\section{Sample preparation}

At the end of the 14-day experimental period, the blood samples were collected from eye medial canthus of each rat while the animal was anaesthetized with ether. Blodd samples were collected in to the tubes containing no anticoagulant. Sera were separated via cold centrifugation at $3000 \times \mathrm{g}$ for 10 minutes at $4^{\circ} \mathrm{C}$. Chol, TG, LDL and HDL concentrations were determined using a commercial diagnostic kits provided by Pars Azmon Co., Iran.

\section{Statistical analysis}

The data obtained in this study were analyzed using ANOVA and LSD's test for multiple comparisons. Statistical significance was determined based on the $\mathrm{p}$ value being set at 0.05 . SPSS, Version 15.0 was used to conduct statistical analysis.

\section{Result}

\section{The Effect of MP pigments on serum Chol level}

After 2-weeks period of treatment, blood factors were determined. Table 2 shows the alterations in sera Chol levels. Comparing the treated animals with the control ones, the result indicate that Chol level in rats sera reduced down to $61.6 \%$ and $35.5 \%$ influenced by use of $25 \%$ and $100 \%$ of Native MP pigments respectively. In rats treated with $25 \%$ and $100 \%$ standard pigment compare with the control animals, cholesterol level was also reduced down to $31 \%$ and $28.9 \%$ respectively. As demonstrated in Table 2 and Figure 1, the overall serum Chol level was significantly lower in the groups treated with both native and standard MP pigments, but the native pigment was more effective in reducing Chol level than the standard strain.

\section{The Effect of MP pigments on serum TG level}

Blood TG level was decreased down to $56.2 \%$ and $32.5 \%$ under the influence of treating the animals with $25 \%$ and $100 \%$ of native MP pigment respectively. In rats treated with $25 \%$ and $100 \%$ standard pigment, TG levels were reduced to $48.8 \%$ and $21.4 \%$ respectively compared with the control animals. Thus, the standard pigment was more effective in decreasing blood TG levels (Table 3 and Figure 2).

\begin{tabular}{|l|l|}
\hline Groups & Diet \\
\hline Control & Tap water \\
\hline $\mathbf{1}$ & $25 \%$ red pigment concentration(Native) \\
\hline $\mathbf{2}$ & $100 \%$ red pigment concentration (Native) \\
\hline $\mathbf{3}$ & $25 \%$ red pigment concentration (STD) \\
\hline $\mathbf{4}$ & $100 \%$ red pigment concentration (STD) \\
\hline
\end{tabular}

Native (M.purpureus isolated in the microbial Collection of the University of Isfahan), STD (M.purpureus DSM1603).

Table 1 : Drink Intake of Experimental Rats.

\begin{tabular}{|l|l|l|l|l|}
\hline & & MD $^{1}$ & MD $^{2}$ & $p$ \\
\hline Groups & Dose of Pigment & Change \% & & \\
\hline Control & & $+15.5 \%$ & & \\
\hline \multirow{2}{*}{ Native Pigment } & $25 \%$ & $-61.6 \%$ & $77.2 \%$ & $0.000^{*}$ \\
\cline { 2 - 5 } & $100 \%$ & $-35.5 \%$ & $49.1 \%$ & $0.000^{*}$ \\
\hline \multirow{2}{*}{ STD Pigment } & $25 \%$ & $-31 \%$ & $46.5 \%$ & $* 0.000$ \\
\cline { 2 - 5 } & $100 \%$ & $-28.9 \%$ & $44.5 \%$ & $* 0.001$ \\
\hline
\end{tabular}

$\mathrm{MD}^{1}$ : Mean Deference compared to baseline

$\mathrm{MD}^{2}$ : Mean Deference compared to the control group

* Significantly different from control group at the same week $(p<0.05)$

Table 2: The effect of Native and STD Pigment on Cholesterol Concentration of Rats serum compared to the control group. 
Citation: Rezaei M, Roghanian R, Nahvi I, Moshtaghian J (2011) Comparing the Effects of Native and Standard Strains of Monascus Purpureus on Fat Metabolism in Rats. J Bioequiv Availab 3: 258-262. doi:10.4172/jbb.1000096

\section{Effect of MP pigments on serum LDL and HDL levels}

As mentioned earlier, the results of this study indicated that MP pigment has a lowering effect on both serum Chol and TG levels. Considering the fact that LDL is one of the risk factors of atherosclerosis, it is remarkable that under the influence of treatment by MP pigment, blood LDL levels were decreased. It is so important that further investigation might be needed. However, the data indicate $40.6 \%$ and $24.6 \%$ reduction in LDL levels due to treatment doses of $25 \%$ and $100 \%$ of standard pigment respectively. Interestingly, the native pigment was more effective in this regard causing $43.2 \%$ and $25.4 \%$ in

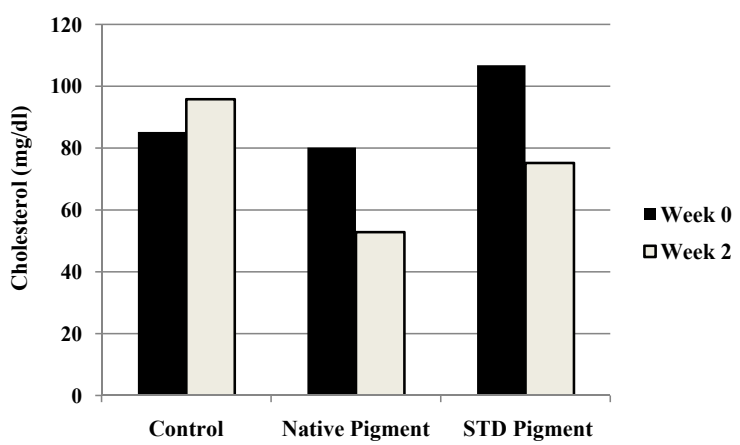

Week 0, baseline; Week 2, post-treatment

Figure 1: The effect of Native and STD pigment on Cholesterol concentration of rat's serum in comparison with the control group.

\begin{tabular}{|l|l|l|l|l|}
\hline & & MD & MD $^{2}$ & $p$ \\
\hline Groups & Dose of Pigment & Change $\%$ & & \\
\hline Control & & $+23.2 \%$ & & \\
\hline \multirow{2}{*}{ Native Pigment } & $25 \%$ & $-56.2 \%$ & $79.4 \%$ & $0.000 *$ \\
\cline { 2 - 5 } & $100 \%$ & $-32.5 \%$ & $55.7 \%$ & $0.000 *$ \\
\hline \multirow{2}{*}{ STD Pigment } & $25 \%$ & $-48.8 \%$ & $72 \%$ & $* 0.000$ \\
\cline { 2 - 5 } & $100 \%$ & $-21.4 \%$ & $44.6 \%$ & $* 0.000$ \\
\hline
\end{tabular}

$M D^{1}$ : Mean Deference compared to baseline

$\mathrm{MD}^{2}$ : Mean Deference compared to the control group

* Significantly different from control group at the same week $(p<0.05)$

Table 3: The effect of Native and STD Pigment on TG Concentration of Rats serum compared to the control group.

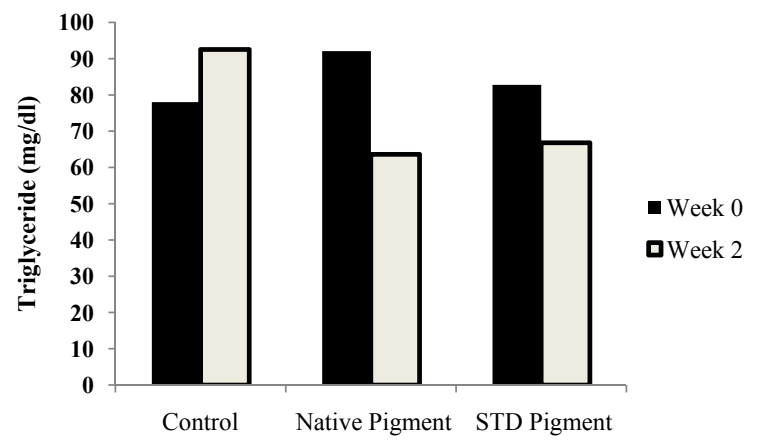

Figure 2: The effect of Native and STD pigment on Triglyceride concentration of rat's serum in comparison with the control group. Week 0 , baseline; Week 2 , post-treatment.

\begin{tabular}{|l|l|l|l|l|}
\hline & & MD & MD & p \\
\hline Groups & Dose of Pigment & Change \% & & \\
\hline Control & & $+20.8 \%$ & & \\
\hline Native Pigment & $25 \%$ & $-43.2 \%$ & $64 \%$ & $\mathbf{0 . 0 0 0}$ \\
\hline STD Pigment & $100 \%$ & $-25.4 \%$ & $46.2 \%$ & $\mathbf{0 . 0 0 7}$ \\
\hline "0.001 & $25 \%$ & $-40.6 \%$ & $61.4 \%$ & $* 0.001$ \\
\hline "0.008 & $100 \%$ & $-24.6 \%$ & $45.4 \%$ & $* \mathbf{0 . 0 0 8}$ \\
\hline
\end{tabular}

$M D^{1}$ : Mean Deference compared to baseline

$\mathrm{MD}^{2}$ : Mean Deference compared to the control group

* Significantly different from control group at the same week $(p<0.05)$

Table 4: The effect of Native and STD Pigment on LDL Concentration of Rats serum compared to the control group.

\begin{tabular}{|l|l|l|l|l|}
\hline & & MD & MD $^{\mathbf{2}}$ & $\mathbf{p}$ \\
\hline Groups & Dose of Pigment & Change \% & & \\
\hline Control & & $-32 \%$ & & \\
\hline \multirow{2}{*}{ Native Pigment } & $25 \%$ & +46.9 & $78.9 \%$ & $\mathbf{0 . 0 0 0}$ \\
\cline { 2 - 5 } & $100 \%$ & $+35.7 \%$ & $67.7 \%$ & $\mathbf{0 . 0 0 1}$ \\
\hline \multirow{2}{*}{ STD Pigment } & $25 \%$ & $+40.2 \%$ & $72.3 \%$ & $* 0.000$ \\
\cline { 2 - 5 } & $100 \%$ & $+26.1 \%$ & $58.1 \%$ & $* 0.003$ \\
\hline
\end{tabular}

$M D^{1}$ : Mean Deference compared to baseline

$\mathrm{MD}^{2}$ : Mean Deference compared to control group

* Significantly different from control group at the same week $(p<0.05)$

Table 5: The effect of Native and STD Pigment on HDL Concentration of Rats serum compared to the control group.

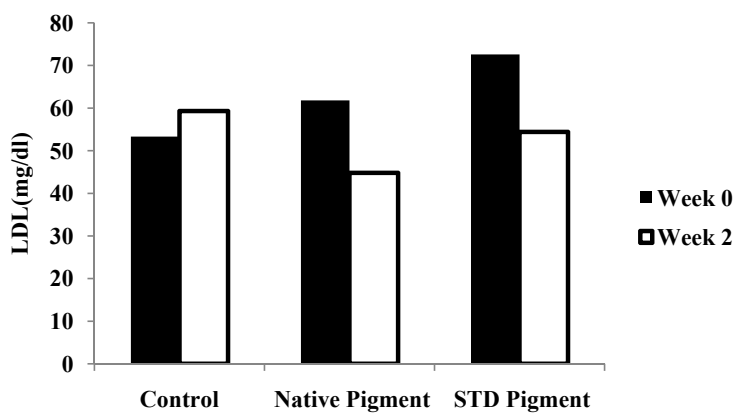

Figure 3: The effect of Native and STD pigment on LDL concentration of rat's serum in comparison with the control group. Week 0 , baseline; Week 2 , posttreatment.

blood LDL levels due to treatments with $25 \%$ and $100 \%$ of doses (Table 4). It is well established that increased blood HDL level is beneficial to human health through reducing the risk for developing cardiovascular diseases. Increases of $40.2 \%$ and $26.1 \%$ in HDL levels were observed under the influence of $25 \%$ and $100 \%$ doses of standard pigment respectively. Remarkably, the native pigment was more effective and increases of $46.9 \%$ and $35.7 \%$ were observed due to treatment of $25 \%$ and $100 \%$ doses respectively (Table 5 and Figure 4).

The ratio of HDL to LDL levels and the Atherogenic Index were other determined criteria (Table 6 and Table 7) which are commonly used to evaluate the efficiency of hypolipidemic medications. If the ratio was high, then the content of HDL had a much higher percentage in total Chol level. I other words, LDL level or the atherosclerotic risk factor was lowered. With this consideration, the statistical analysis of the data indicated that in rats fed with MP pigments blood HDL 
Citation: Rezaei M, Roghanian R, Nahvi I, Moshtaghian J (2011) Comparing the Effects of Native and Standard Strains of Monascus Purpureus on Fat Metabolism in Rats. J Bioequiv Availab 3: 258-262. doi:10.4172/jbb.1000096

\begin{tabular}{|l|l|l|l|l|l|l|}
\hline & & HDL-C/LDL-C & HDL-C/LDL-C & MD & MD \\
\hline Groups & Dose of Pigment & Week 0 & Week 2 & Change \% \\
\hline Control & & 0.43 & 0.26 & $58.7 \%$ & \\
\hline \multirow{2}{*}{ Native Pigment } & $25 \%$ & 0.16 & 0.65 & $72 \%$ & $130.8 \%$ \\
\cline { 2 - 6 } & $100 \%$ & 0.49 & 1.08 & $51.8 \%$ & $110.6 \%$ \\
\hline \multirow{2}{*}{ STD Pigment } & $25 \%$ & 0.25 & 0.79 & $66.3 \%$ & $0.000^{*}$ \\
\cline { 2 - 6 } & $100 \%$ & 0.28 & 0.60 & $44.3 \%$ & $125.1 \%$ \\
\hline
\end{tabular}

MD': Mean Deference compared to baseline

$\mathrm{MD}^{2}$ : Mean Deference compared to control group

Week 0, baseline; Week 2, post-treatment

*Significantly different from control group at the same week $(p<0.05)$

Table 6: HDL-C/LDL-C changes in pigment treated groups compared to the control group.

\begin{tabular}{|c|c|c|c|c|c|c|}
\hline & & $\mathrm{Al}^{1}$ & $\mathrm{Al}^{2}$ & $\mathrm{MD}^{1}$ & $\mathrm{MD}^{2}$ & $\mathrm{p}$ \\
\hline Groups & Dose of Pigment & Week 0 & Week 2 & Change \% & & \\
\hline Control & & 4.36 & 5.70 & $82 \%$ Increase & & \\
\hline \multirow{2}{*}{ Native Pigment } & $25 \%$ & 10 & 0.90 & 89.57\% Decrease & $171.60 \%$ & $0.000 *$ \\
\hline & $100 \%$ & 2.33 & 0.37 & $94.65 \%$ Decrease & $\% 176.68$ & $0.000 *$ \\
\hline \multirow{2}{*}{ STD Pigment } & $25 \%$ & 4 & 0.89 & $78.5 \%$ Decrease & $160.55 \%$ & $* 0.000$ \\
\hline & $100 \%$ & 4.77 & 2.31 & $54.94 \%$ Decrease & $136.97 \%$ & $* 0.000$ \\
\hline
\end{tabular}

$\mathrm{Al}^{1}$ : Atherogenic Index at Week 0 (baseline)

$\mathrm{Al}^{2}$ : Atherogenic Index at Week 2(post-treatment)

$\mathrm{MD}^{1}$ : Mean Deference compared to baseline

MD2: Mean Deference compared to the control group

*Significantly different from control group at the same week $(p<0.05)$

Table 7: Atherogenic Index in Pigment treated groups compared to the control group.

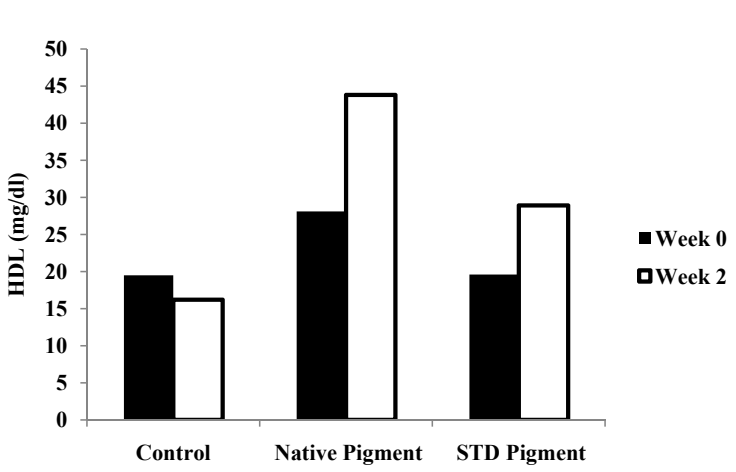

Figure 4: The effect of Native and STD pigment on HDL concentration of rat's serum in comparison with the control group. Week 0 , baseline; Week 2, posttreatment.

levels were significantly increased while their blood LDL levels were decreased $(\mathrm{p}<0.05)$

\section{Discussion}

It was earlier reported that that red mold rice caused significant decreases in LDL, Chol, TG levels [29]. In this study, two weeks of treatment with native MP pigment resulted in significant reductions in LDL (25.4\%), Chol (35.5\%) and TG (32.5\%) levels from the baseline. As shown in the results, pigment of Native MP significantly reduced TC, TG, and LDL-C levels and increased the HDL-C level. The results of the present study showed that Monascus pigment significantly decreased TC and TG levels at the fourth-fold dosage.

Feeding rats with Monascus pigment at fourth -fold dosage administered at a lower dosage did indeed have a remarkable effect of cholesterol-lowering action as compared with the higher dosage of Monascus pigment.

There was no statistically significant difference percentage of decrease in TG, LDL and HDL levels after treatment between Native and Standard pigment.

In addition, Rats taking pigment had an increase in HDL (good) cholesterol and a decrease in LDL (bad) cholesterol with the supplement.

The result of this study showed that adding red pigment containing broth medium fermented by $\mathrm{M}$. purpureus (25\% and $100 \%$ concentrations) into Rat' diet could significantly decrease TC, TG, and LDL levels. The particular dosage of pigment to be administered should be effective to reduce LDL-cholesterol concentration and to increase HDL-cholesterol concentration in the blood, and will depend on the route of administration. The addition of suspension produced through submerged fermentation of Monascus into Rat' diet not only increases HDL-C concentration but also, the ratio of HDL-C/LDL-C for the experimental groups was higher than that of the control group.

Since possesses the ability to lower cholesterol level. It has been developed into functional health food for human dietary supplement.

Previous studies were similar to this study in showing a marked effect of the pigment containing supplement on cholesterol concentrations. Pigment used in this study confirms Monascus purpureus red rice that it had positive effects on plasma lipids. However, there were differences in the route of pigment administration. Furthermore, pigment containing red yeast rice is only $0.3 \%$ [12] and less than pigment containing of suspension which used in this study. Red rice was produced through solid state fermentation but in the present study we used different natural pigment preparation through submerged fermentation. 
Citation: Rezaei M, Roghanian R, Nahvi I, Moshtaghian J (2011) Comparing the Effects of Native and Standard Strains of Monascus Purpureus on Fat Metabolism in Rats. J Bioequiv Availab 3: 258-262. doi:10.4172/jbb.1000096

Despite the advantages of using RYR, the presence of citrinin in the products has a negative effect on its acceptance by the people. Hsieh and Pan found that the citrinin content in red mold rice in a Taiwan market ranged from 0.1 to $122 \mathrm{ppm}$ [30]. Therefore, the reduction of citrinin draws a lot of attention by researchers throughout the world. However, in this study by using optimization of the culture medium, no adverse effect was observed in the treated animal comparing with the control group. It is worthy to notify that a native strain was used in this experiment. In further studies we will try to use genetic engineering techniques to obtain mutants with lower citrinin producers.

\section{Acknowledgment}

This research was supported by the grants received from the University of Isfahan. The authors are grateful for the financial support.

\section{References}

1. Hopwood DA, Sherman DH (1990) Molecular genetics of polyketides and its comparison to fatty acid biosynthesis. Ann Rev Gen 24: 37-66.

2. Chen MH, Johns MR (1993) Effect of pH and nitrogen source on pigment production by Monascus purpureus. Appled Microbiology Biotechnology 40: 132-38.

3. Pattanagul P, Pinthong R, Phianmongkhol A, Leksawadi N (2007) Review of Angkak Production (Monascus purpureus). Chiang Mai J Sci 2007; 34: 319-28.

4. Lee CL, Tsai TY, Wang JJ, Pan TM (2006) In vivo hypolipidemic effects and safety of low dosage Monascus powder in a hamster model of hyperlipidemia. Appl Microbiol Biotechnol 70: 533-540.

5. Yasukawa K, Takahashi M, Natori S, Yamazaki M, Takeuchi M, et al. (1994) Azaphilones inhibit tumor promotion by 12-O-tetradecanoylphorbol-13-acetate in two-stage carcinogenesis in mice. Oncology 51:108-112.

6. Martínková L, Patáková-Jůzlová P, Krent V, Kucerová Z, Havlícek V, et al (1999) Biological activities of oligoketide pigments of Monascus purpureus. Food Addit. Contam 16: 15-24.

7. Chang JC, Wu MC, Liu IM, Cheng JT (2006) Plasma glucose-lowering action of Hon-Chi in streptozotocin-induced diabetic rats. Horm Metab Res 38: 76-81.

8. Aniya Y, Ohtani II, Higa T, Miyagi C, Gibo H, et al. (2000) Dimerumic acid as an antioxidant of the mold, Monascus anka. Free Radical Biol Med 28: 999-1004.

9. Wu GF, Wu XC (2000) Screening DPPH radical scavengers from Monascus sp. Wei Sheng Wu Xue Bao. 40: 394-349.

10. Dhale MA, Divakar S, Kumar SU, Vijayalakshmi G (2007) Isolation and characterization of dihydromonacolin-MV from Monascus purpureus for antioxidant properties. Appl Microbial Biotechnol 73: 1197-1202.

11. Korantzopoulos P, Kokkoris S (2004) The antioxidant effects of statins may extend beyond atherosclerosis: potential benefit for atrial fibrillation and heart failure. Atherosclerosis 175:187.

12. Endo A (1979) Monacolin K, a new hypocholesterolemic agent produced by Monascus species. Antibiot (Tokyo). 32: 852-854.

13. Bach TJ (1986) Hydroxymethylglutaryl-CoA reductase, a key enzyme in phytosterol synthesis. Lipids 21: 82-88.

14. Endo A, Hasumi K. Mevinic acids. In: Anke, T. (Ed.), Fungal Biotechnol. Chapman \& Hall, Weinheim 1997; 162-172.

15. Wang J, Lu Z, Chi J, Wang W, Su M, et al. (1997) A multi-center clinical trial of the serum lipid lowering effects of a Monascus purpureus (red yeast) rice preparation from traditional Chinese medicine. Current Therapeutic Research 58: 964-978

16. Li C, Zhu Y, Wang Y, Zhu JS, Chang J, et al. (1998) Monascus purpureus fermented rice (red yeast rice): a natural food product that lowers blood cholesterol in animal models of hypercholesterolemia. Nutrition Research 18 71-78

17. Kennedy J, Auclair K, Kendrew SG, Park C, Vederas JC, et al. (1999) Modulation of polyketide synthase activity by accessory proteins during lovastatin biosynthesis. Science 284: 1368-1372.
18. Heber D, Lembertas A, Lu QY, Bowerman S, Go VL (2001) An analysis of nine proprietary Chinese red yeast rice dietary supplements: implications of variability in chemical profile and contents. J Altern Complement Med 7: 133139.

19. Francis FJ (1987) Lesser-known food colorants. Food Technol 41: 62-68.

20. Frink-Gremmels J, Dresl J, Leistner J (1991) Use of Monascus extracts as an alternation to nitrite meat products. Kleischwirtsch 71: 329-331.

21. Fabre CE, Santerre AL, Lore MO, Banerian RO, Parcilleux AR, et al. (1993) Production and food applications of the red pigments of Monascus ruber. Journal of Food Science 58: 1099-1102.

22. Cheng JH, Ockerman AW (1998) Eflects of anka rice, nitrite, and phosphate on warmed-over flavor and palatability characteristics in roast beef. Meat Sci 49: 65-78.

23. Bakosova A, Mate D, Laciakova A, Pipova M (2001) Utilization of Monascus purpureus in the production of foods of animal origin. Bull. Vet. Inst. Pulawy 45: 111-116.

24. Yu CC, Wang JJ, Lee CL, Lee SH, Pan TM (2008) Safety and Mutagenicity Evaluation of Nanoparticulate Red Mold Rice. J. Agric. Food Chem 56: 11038 11048.

25. Sanae P, Fernanda S, Vahan B(2005) Concentration determination of extracellular and intracellular red pigments produced by Monascus sp. Braz. arch. biol. Technol 48: 43-49.

26. Panagou EZ, Skandamis PN, Nychas GJE (2005) Use of gradient plates to study combined effect of temperature, $\mathrm{pH}$, and $\mathrm{NaCl}$ concentration on growth of Monascus rubber van Tieghem, an ascomycetes fungus isolated from Green table olives. Appl.environ. Microbiol 71: 392-399.

27. Wang YZ, Ju XL, Zhou YG (2005) The variability of citrinin production in Monascus type cultures. Food microbiol 22: 145-148.

28. Su YC, Wang JJ, Lin TT, Pan, TM (2003) Production of the secondary metabolites $\mathrm{Y}$-aminobutyric acid and monacolin $\mathrm{K}$ by Monascus. $\mathrm{J}$ Ind Microbio Biotechnol 30: 41-46.

29. Heber D, Yip I, Ashley JM, Elashoff DA, Elashoff RM, et al. (1999) Cholesterollowing effects of a proprietary Chinese red-yeast-rice dietary supplement. Am J Clin Nutr 69: 231-6.

30. Hsieh YT, Pan TM (2002)The analytic methods and assays for secondary metabolites of Monascus products. J Biomass Energy Soc China 21: 63-71. 